\title{
Signal Remodulation Ring WDM Passive Optical Network with Rayleigh Backscattering Interferometric Noise Mitigation
}

\author{
C. H. Yeh, Member, IEEE, and C. W. Chow, Member, IEEE
}

\begin{abstract}
We propose and demonstrate a novel and simple signal remodulation ring-based wavelength division multiplexed passive optical network (WDM-PON) scheme with the capability to mitigate the Rayleigh backscattering ( $R B)$ beat noise in colorless signal remodulation operation. The effects of $\mathrm{RB}$ can be avoided since the backscattering effects and upstream signals travel in opposite directions. Here, $10 \mathrm{~Gb} / \mathrm{s}$ DPSK downstream is used to launch into reflective optical semiconductor amplifier (RSOA)-based optical network unit (ONU) for signal remodulation at 1.25 or $2.5 \mathrm{~Gb} / \mathrm{s}$ on-off keying (OOK). Moreover, the network performance has also been analyzed and discussed.
\end{abstract}

Index Terms-Rayleigh backscattering, WDM, passive optical network (PON), DPSK, OOK, sensitivity.

\section{INTRODUCTION}

$\mathbf{S}$ INCE the demand for broadband services is increasing rapidly, the last mile data access network must provide high data rate in a more economic way. Time division multiplexed passive optical network (TDM-PON) and wavelength division multiplexed PON (WDM-PON) have been widely developed for the access networks [1], [2]. Meanwhile WDMPON technologies can also provide symmetric bandwidth connections at very high data rates, in the 1-10 Gbps range per end-user [3]. Here, new "colorless" ONU designs can be leveraged to help reduce WDM-PON costs as well, i.e., by obviating the need for wavelength-specific lasers using technologies such as reflective semiconductor optical amplifier (RSOA) or Fabry-Perot laser diodes (FP-LD) [4]. However, colorless ONU can lead to Rayleigh backscattering (RB) interferometric beat noise, which is produced at the receiver at central office $(\mathrm{CO})$. The RB beat noise could degrade the received signal to noise ratio (SNR) and reduce the received sensitivity [5]. To avoid and mitigate the RB noise in WDMPONs, several technologies have been developed and reported, such as using phase and bias-current dithering, advanced modulation formats, and wavelength shifting [6]-[9]. However, the above methods either need to use complicated modulation schemes or employ dithering techniques to broaden the upstream wavelength, which would decrease the dispersion tolerance. In addition, these proposed techniques are most suited for use in tree-architecture WDM-PONs. In this paper, a ringarchitecture WDM-PON with RB noise mitigation is proposed and experimentally investigated. A new module design in each

Manuscript received September 29, 2010. The associate editor coordinating the review of this letter and approving it for publication was A. Shami.

C. H. Yeh is with the Information and Communications Research Laboratories, Industrial Technology Research Institute (ITRI), Chutung, Hsinchu 31040, Taiwan (e-mail: yeh1974@gmail.com).

C. W. Chow is with the Department of Photonics and the Institute of Electro-Optical Engineering, National Chiao Tung University, Hsinchu 30010, Taiwan.

Digital Object Identifier 10.1109/LCOMM.2011.082611.101838

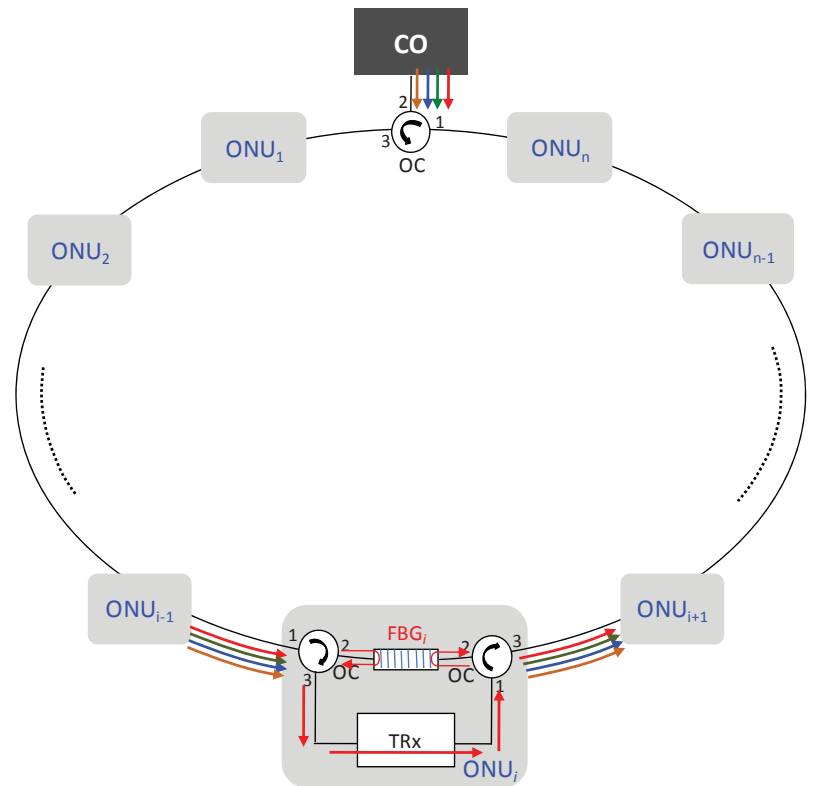

Fig. 1. Proposed ring-architecture WDM-PON system to mitigate the Rayleigh backscattering noise.

RSOA-based ONU is used to produce the downstream and upstream traffic in the two different directions and advanced modulation techniques are not required to avoid RB noise. Moreover, the $10 \mathrm{~Gb} / \mathrm{s}$ differential phase shift keying (DPSK) downstream and $1.25 \mathrm{~Gb} / \mathrm{s}$ (or $2.5 \mathrm{~Gb} / \mathrm{s}$ ) remodulated on-off keying (OOK) upstream traffic are achieved in the proposed PON system.

\section{EXPERIMENT AND DISCUSSION}

Fig. 1 illustrates the proposed ring-based WDM-PON to mitigate RB beat noise. The WDM downstream signals will transmit via optical circulator (OC) into each ONU in counterclockwise (CCW) direction. Here, each ONU is consisted of two OCs, an optical transceiver (TRx), and a fiber Bragg grating $(\mathrm{FBG})$, which is used to filter and reflect the corresponding downstream wavelength into TRx for upstream signal remodulation, as shown in Fig. 1. Hence, the remodulated upstream signal also will be reflected by FBG and transmit through the same $\mathrm{CCW}$ direction into the $\mathrm{CO}$. Based on the proposed network architecture, the downstream and upstream wavelengths could propagate in two different directions to mitigate RB noise.

Fig. 2 shows the experimental setup for the proposed ringbased WDM-PON. In the CO, we use a $1550.1 \mathrm{~nm}$ continuouswave $(\mathrm{CW})$ light to launch into a $\mathrm{LiNbO}_{3}$ phase modulator $(\mathrm{PM})$ to generate the $10 \mathrm{~Gb} / \mathrm{s}$ DPSK downstream signal 


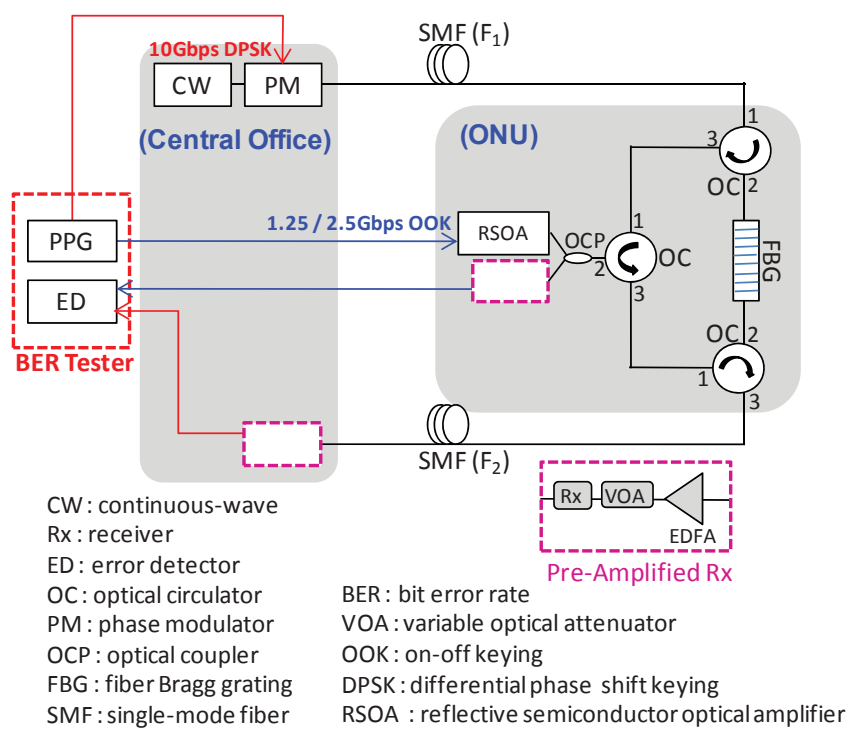

Fig. 2. Experimental setup for the proposed ring-based WDM-PON system.

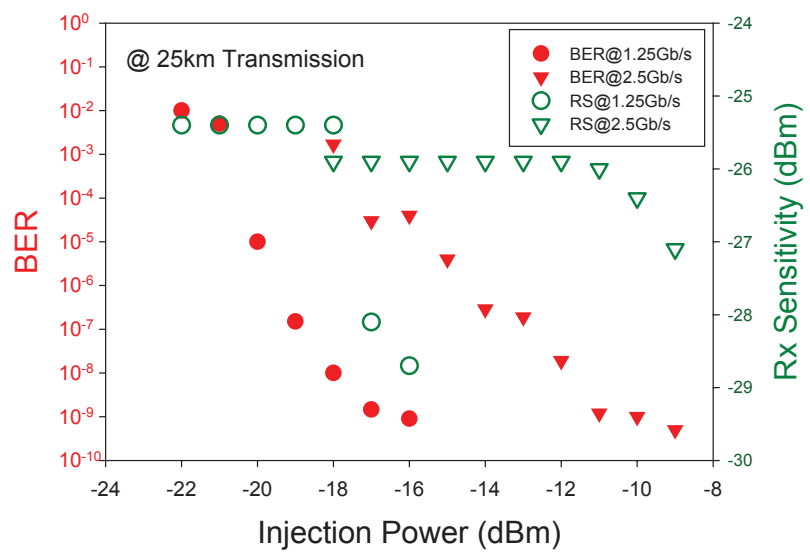

Fig. 3. Measured performances of BER and Rx sensitivity under different injected power levels for the RSOA-based ONU in $25 \mathrm{~km}$ fiber transmission.

by encoding with a differentially precoded nonreturn-to-zero (NRZ) data stream with $2^{31}-1$ pattern length. Here, the ONU is constructed by three OCs, a FBG, a RSOA, a 50 : 50 optical coupler $(\mathrm{OCP})$, a receiver $(\mathrm{Rx})$ and a delay interferometer (DI). The reflection and central wavelength of FBG used are $99.5 / \%$ and $1550.16 \mathrm{~nm}$, respectively. The operation current of the RSOA is operated at $80 \mathrm{~mA}$. The two single-mode fibers (SMFs) of $F_{1}$ and $F_{2}$ are the downstream and upstream transmission paths, respectively, as seen in Fig. 2. The total maximum fiber lengths of $F_{1}$ and $F_{2}$ are around $25.5 \mathrm{~km}$ for downstream and upstream traffic. This experimental setup basically reflects an all-optical signal re-modulation scenario. Since the downstream DPSK signal is phase modulated with constant intensity, the upstream OOK signal can be easily remodulated onto the DPSK signal.

In the experiment, the combinations of the $\mathrm{F}_{1}$ and $\mathrm{F}_{2}$ should be $0.5 \mathrm{~km}+25 \mathrm{~km}, 10 \mathrm{~km}+10 \mathrm{~km}$ and $25 \mathrm{~km}+0.5 \mathrm{~km}$, respectively, for downstream and upstream measurements. The downstream DPSK signal will be reflected by the FBG and launched into the RSOA via an OC, and the residual 50/\% optical power can be received by an optically pre-amplified receiver. Namely, this receiver consists of a variable optical attenuator (VOA), erbium-doped fiber amplifier (EDFA), a delay interferometer (DI) for DPSK demodulation, and a 10$\mathrm{Gb} / \mathrm{s}$ PIN. The rest of the downstream power is launched into the RSOA to generate the upstream signal. The upstream signal of RSOA can be remodulated at 1.25 and $2.5 \mathrm{~Gb} / \mathrm{s}$ OOK format with the NRZ pseudo random binary sequence (PRBS) with pattern length of $2^{31}-1$. Carefully note that the data rate is limited by the RSOA and that the proposed scheme can achieve higher speeds.

To investigate the relationship of downstream injectedpower level and output performance of remodulated upstream signal first, different powers of DPSK downstream signal are injected into the RSOA for upstream remodulation. Fig. 3 shows the measured bit-error rates (BERs) and receiver sensitivities of the upstream signal under different injected-power levels at 1.25 and $2.5 \mathrm{~Gb} / \mathrm{s}$ OOK modulation, respectively, in $25 \mathrm{~km}$ single-mode fiber (SMF) transmission. Fig. 3 shows that improved BER performance depends upon larger injection powers. Besides, in order to achieve the 1.25 and $2.5 \mathrm{~Gb} / \mathrm{s}$ signal remodulation at the BER of $10^{-9}$, the downstream injection must be 16 and $10 \mathrm{dBm}$ and the $\mathrm{Rx}$ sensitivity are measured at 28.7 and $26.4 \mathrm{dBm}$ after $25 \mathrm{~km}$ SMF link, respectively, as shown in Fig. 3. We can observe in Fig. 3 that when the injected power is low, the BER and receiver sensitivity are very stable. When the injected power increases from $18 \mathrm{dBm}$ (at $1.25 \mathrm{~Gb} / \mathrm{s}$ ) and $12 \mathrm{dBm}$ (at $2.5 \mathrm{~Gb} / \mathrm{s}$ ), the BER and the receiver sensitivity decrease and finally saturate. We then measure the $10 \mathrm{~Gb} / \mathrm{s}$ DPSK downstream signal at the ONU for fiber lengths $F_{1}$ of $0.5,10$ and $25 \mathrm{~km}$. For the upstream traffic, the RSOA is injected by the residual power $(90 / \%)$ of downstream signal and can be directly modulation at 1.25 and $2.5 \mathrm{~Gb} / \mathrm{s}$ by NRZ PRBS format, for F2 fiber length of $0.5,10$ and $25 \mathrm{~km}$. As a result, Fig. 4(a) and Fig. 4(b) show the measured BER performances for the downstream and upstream traffic under different fiber lengths link. Fig. 4(a) shows the $10 \mathrm{~Gb} / \mathrm{s}$ downstream power penalty of $; 0.2$ $\mathrm{dB}$ at a BER of 10-9 under different F1 lengths. Meanwhile the measured maximum power penalties at $1.25 \mathrm{~Gb} / \mathrm{s}$ and 2.5 $\mathrm{Gb} / \mathrm{s}$ are $1 \mathrm{~dB}$ and $0.8 \mathrm{~dB}$ respectively, as shown in Fig. 4(b). We believe the slight difference in power penalty may be due to experimental error. We can conclude that the power penalties at $1.25 \mathrm{~Gb} / \mathrm{s}$ and $2.5 \mathrm{~Gb} / \mathrm{s}$ are similar. Moreover, the inserts in Figs. 4(b) and 4(b) show the corresponding eye diagrams in DPSK and OOK formats. Overall, the measured eyes are clear and wide opening at back-to-back and 25 $\mathrm{km}$ transmission. Since the downstream DPSK signal and upstream OOK signals are traveling in two different fibers, the Rayleigh backscattering can be avoided. We can observe that the upstream signals are not affected by the RB noise since the proposed architecture allows the back-reflected RB noise and the upstream signal travel in opposite directions. Finally, we have taken into account the signal losses of optical fibers at $0.5 \mathrm{~km}, 10 \mathrm{~km}$ and $25 \mathrm{~km}$ in Figs. 4(a) and 4(b). These locations could represent the remote nodes (RNs) located at the near end and far end of the $25 \mathrm{~km}$ ring network. And we can achieve error-free transmission as shown in Figs. 4(a) and 4(b). Although we have just used one RN in the experiment (as illustrated in Fig. 2), by considering the insertion losses 

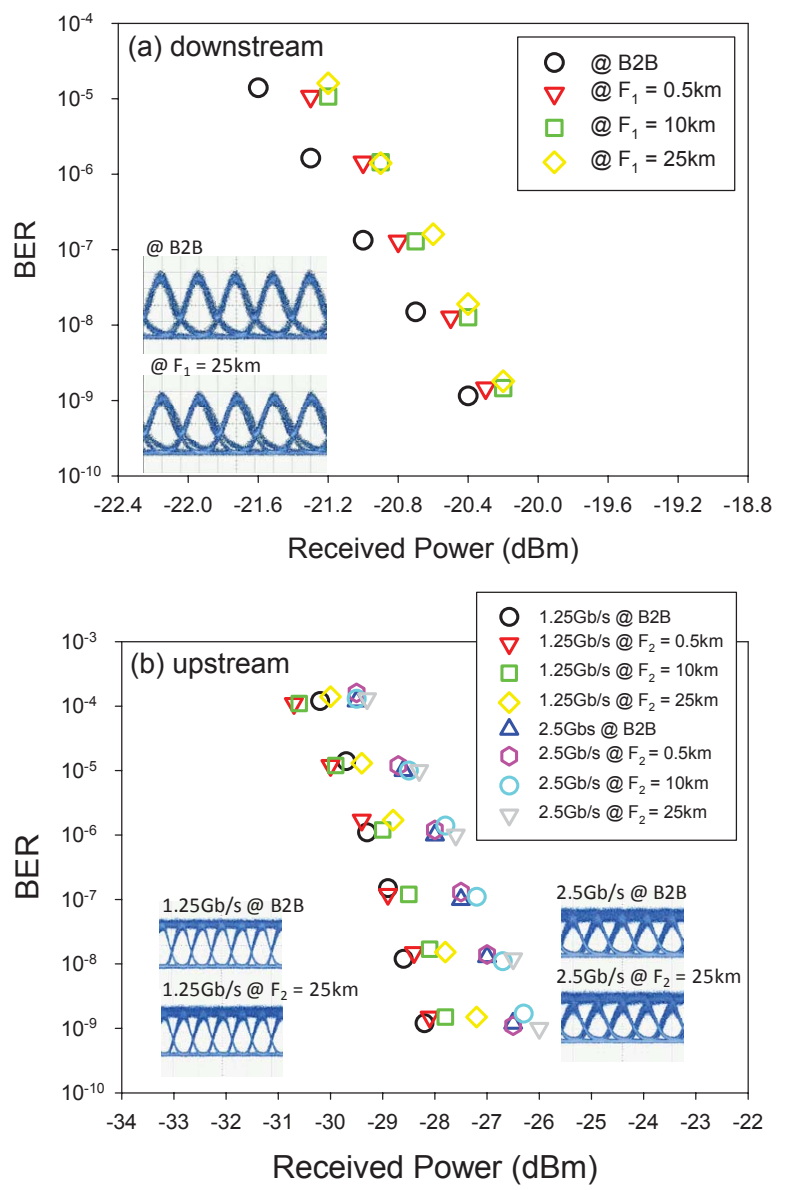

Fig. 4. Measured BER performances for the (a) downstream and (b)upstream traffic under different fiber lengths link. The inserts are corresponding eye diagrams.

for FBG pass-band and the two optical circulators in each RN are $0.1 \mathrm{~dB}$ and $1 \mathrm{~dB}$ respectively, we can calculate that each transmitted wavelength channel will experience an insertion loss of $1.1 \mathrm{~dB}$ at each $\mathrm{RN}$. If the power budget for the whole network is much higher than the receiver sensitivities shown in Figs. 4(a) and 4(b), we believe that the insertion loss for the $\mathrm{RN}$ will not affect the signal performance. Here, we assume the optical power of the downstream signal and the receiver sensitivity (without using an optical pre-amplified receiver) are $7 \mathrm{dBm}$ and $15 \mathrm{dBm}$ respectively. And the insertion losses of each RN, $25 \mathrm{~km} \mathrm{SMF}$ and the 12 optical coupler before the RSOA are 1.1, 5 and $3 \mathrm{~dB}$ respectively, as illustrated in Fig. 1. Hence, eight RNs can be supported in the proposed access network when nearly $10 \mathrm{dBm}$ optical injection power can be launched into the RSOA (error-free upstream signal generation as shown in Fig. 3) at eighth ONU.

\section{CONCLUSIONS}

We have proposed and experimentally investigated a ringbased signal remodulation WDM-PON system to mitigate RB beat noise. In this proposed network, a new ONU is designed to generate two propagating directions for downstream and upstream traffic. Hence, RB can be suppressed in the proposed system since the RB and the upstream channel are transmitting in opposite directions. Moreover, $10 \mathrm{~Gb} / \mathrm{s}$ DPSK downstream traffic and $2.5 \mathrm{~Gb} / \mathrm{s}$ OOK upstream remodulation can be achieved in the WDM access network.

\section{REFERENCES}

[1] H. Shinohara, "Overview of Japanese FTTH market and NTT's strategies for entering full-scale FTTH era," in APOC 2007, invited paper, paper FTTH2.1.

[2] “10Gb/s Ethernet Passive Optical Network," IEEE 802.3av, Sep. 2009.

[3] C. H. Yeh, C. W. Chow, and S. Chi, "Using $10 \mathrm{~Gb} / \mathrm{s}$ remodulation DPSK signal for self-restored colorless WDM-PON system," Opt. Fiber Technol., vol. 15, pp. 274-278, 2009.

[4] C. H. Yeh, C. W. Chow, F. Y. Shih, C. H. Wang, Y. F. Wu, and $\mathrm{S}$. Chi, "Wavelength tunable laser for signal remodulation in WDM access networks using DPSK downlink and OOK uplink," IEEE Photon. Technol. Lett., vol. 21, pp. 1710-1712, 2009.

[5] Z. Li, Y. Dong, Y. Wang, and C. Lu, "A novel PSK-Manchester modulation format in $10-\mathrm{Gb} / \mathrm{s}$ passive optical network system with high tolerance to beat interference noise," IEEE Photon. Technol. Lett., vol. 17 , pp. 1118-1120, 2005.

[6] P. J. Urban, A. M. J. Koonen, G. D. Khoe, and H. de Waardt, "Rayleigh backscattering-suppression in a WDM access network employing a reflective semiconductor optical amplifier," in Proc. Symposium IEEE/LEOS Benelux Chapter, pp. 147-150, 2007.

[7] C. W. Chow, G. Talli, and P. D. Townsend, "Rayleigh noise reduction in 10-Gb/s DWDM-PONs by wavelength detuning and phase-modulationinduced spectral broadening," IEEE Photon. Technol. Lett., vol. 19, pp. 423-425, 2007.

[8] A. Chowdhury, H.-C. Chien, M.-F. Huang, J. Yu, and G.-K. Chang, "Rayleigh backscattering noise-eliminated 115-km long-reach bidirectional centralized WDM-PON with 10-Gb/s DPSK downstream and remodulated 2.5-Gb/s OCS-SCM upstream signal," IEEE Photon. Technol. Lett., vol. 20, pp. 2081-2083, 2008.

[9] C. H. Wang, C. W. Chow, C. H. Yeh, C. L. Wu, S. Chi, and C. Lin, "Rayleigh noise mitigation using single sideband modulation generated by a dual-parallel MZM for carrier distributed PON," IEEE Photon. Technol. Lett., vol. 22, pp. 820-822, 2010. 